\title{
El servicio de documentación en las organizaciones empresariales
}

\author{
Miguel Ángel del Prado Martínez \\ Federación de Empresarios del Metal de Zaragoza, CEPYME \\ Aragón, CEPYME Zaragoza y Fundación "Cultura y Empresa" \\ Centro de Documentación.
}

\section{1. Resumen}

Se presenta el proyecto de planificación y puesta en funcionamiento del Servicio de Documentación y Archivos de las organizaciones empresariales Federación de Empresarios del Metal de Zaragoza, CEPYME Aragón, CEPYME Zaragoza y la Fundación "Cultura y Empresa", creado en abril de 1999, así como su evolución y retos actuales. Se constata la variada tipología de documentos que requiere una organización empresarial, así como la importancia de una adecuada gestión de la información para el aprovechamiento de las tecnologías de la información, entre las que se resaltan por su utilidad las de trabajo en grupo. La utilización de información de calidad por los miembros de una organización es la que marcará el éxito de su sistema de información.

Palabras clave: Servicios de documentación. Sistemas de información. Organizaciones empresariales.

\section{2. Abstract}

The planning project, implementation, evolution and current challenges of the Documentation Service and Archives of the business organizations Federation of Metal Employers of Zaragoza (Spain), Confederations of the Medium and Small Firms of Aragón and Zaragoza, and the "Culture and Enterprise" Trust, created in April 1999, are presented. The various typologies of documents that such organizations require and the need for an adequate and centralized management of their information is confirmed. The use of information of quality by the members of the organization will point out the success of the information system. The simple use of information technologies disregarding of persons, information characteristics and proper organization can lead to big disappointments.

Palabras clave: Documentation services. Information systems. Business organizations. 


\section{Introducción}

Las organizaciones empresariales Federación de Empresarios del Metal de Zaragoza (FEMZ), Confederación de la Pequeña y Mediana Empresa Aragonesa (CEPYME Aragón), Confederación de la Pequeña y Mediana Empresa de Zaragoza (CEPYME Zaragoza) y Fundación "Cultura y Empresa”, ante los crecientes problemas que sufrían para controlar la documentación que se iba acumulando en su sede, deciden contratar a una persona especializada en gestión documental. De esta manera, a finales de abril del año 1999 ponen en marcha el Servicio de Documentación y Archivos. En este artículo se quiere dar a conocer el proceso seguido en la planificación y puesta en funcionamiento de este servicio, los objetivos que hasta la fecha han sido alcanzados y los retos que en este momento tiene planteados.

\section{Las organizaciones empresariales}

En primer lugar, para conocer el contexto en el que surge el Servicio se señalarán brevemente las características fundamentales de las organizaciones empresariales. Las organizaciones empresariales son entidades asociativas para la representación, defensa y promoción de los intereses económicos y sociales de sus asociados, esto es, de las empresas afiliadas directa o indirectamente (1). Las organizaciones empresariales agrupan a las empresas de un sector de actividad económica o de un territorio determinado, por lo que se diferencianen organizaciones sectoriales y organizaciones intersectoriales territoriales. Las organizaciones sectoriales, de rama o categoría profesional agrupan las empresas que tienen en común un producto o conjunto de productos, un mercado o una profesión. Esta estructura de ramas o sectores comprende un gran número de asociaciones, federaciones o confederaciones de distintos niveles territoriales, desde el nacional hasta el local, y que abarcan desde actividades muy concretas y especializadas hasta grandes sectores con numerosas ramas de actividad. Las organizaciones intersectoriales territoriales agrupan a las organizaciones - ya sean territoriales o sectoriales- de un ámbito territorial. Existen organizaciones intersectoriales de ámbito nacional, autonómico, provincial, comarcal e incluso local. En lo más alto de la estructura empresarial española están CEOE y CEPYME, organizaciones intersectoriales con competencia en asuntos comunes a todas las empresas españolas.

La prestación de servicios a los asociados constituye el eje vertebral de toda organización empresarial. Estos servicios se pueden clasificar en dos tipos. El primer tipo son servicios de representación y defensa de los intereses empresariales. La representación institucional de los empresarios asociados constituye el primer objetivo estatutario de las organizaciones. Mejorar el entorno económico, fiscal, laboral, social, etc., en el que se desarrolla la actividad empresarial es la actividad más propia y característica de las organizaciones empresariales. El segundo tipo

Scire. $8: 2$ (jul.-dic. 2002) 173-188. 
son servicios de prestación de servicios técnico profesionales. Las organizaciones empresariales se han mostrado como magníficos instrumentos de apoyo al desarrollo de las empresas mediante la prestación directa de servicios a las mismas. Entre los servicios técnicos prestados por las organizaciones empresariales están: consultoría y asesoramiento en materias diversas — asuntos jurídicos, laborales, fiscales, económicos, calidad, medio ambiente, prevención de riesgos laborales, nuevas tecnologías-, formación empresarial y profesional, apoyo a las acciones de internacionalización y marketing, servicios que facilitan los vínculos de relación empresarial entre las pequeñas y grandes empresas, servicios que facilitan el acceso de las empresas a la investigación y la innovación, acciones colectivas conseguir productos o servicios que utilizan las empresas en unas condiciones mejores de precio en función del volumen contratado: tarifas telefónicas, postales, comunicación, seguros-, servicios laborales —negociación laboral, asesoramiento legal, orientación profesional, prácticas en empresas, bolsas de trabajo, observatorios del mercado de trabajo-, y servicios de comunicación social. Habitualmente, cuanto más alto se sitúa la organización en la pirámide asociativa, son más genéricos los intereses que defiende y más amplio y variado su ámbito de representación, por lo que mayor es la atención prestada a la función representativa y a los servicios derivados de la misma. En sentido inverso, la organización de base sectorial territorial es habitualmente el centro de prestación de servicios técnicos profesionales directos a la empresa, en particular a las pequeñas y medianas empresas.

La estructura interna de cada organización empresarial depende de su nivel competencial y grado de especialización, pero en líneas generales en todas ellas encontramos unos órganos de gobierno (presidencia, vicepresidencia, asamblea general, comité ejecutivo), unos órganos administrativos encabezados por la secretaría general (administración general y contabilidad) y un conjunto más o menos amplio de departamentos encargados de la prestación de servicios.

Por lo que respecta a las cuatro organizaciones empresariales que nos ocupan (FEMZ, CEPYME Aragón, CEPYME Zaragoza y Fundación "Cultura y Empresa") es importante destacar que son organizaciones que mantienen amplias relaciones, del tal manera que, teniendo su sede en el mismo edificio, algunos de sus departamentos trabajan conjuntamente. No obstante gozan de plena personalidad jurídica, con sus propios órganos de gobierno, con unas funciones y actividades claramente diferenciadas, y con plena autonomía en su funcionamiento.

La Federación de Empresarios del Metal de Zaragoza (FEMZ), fue constituida por los empresarios del metal en el año 1977, para representar y defender los intereses colectivos de las empresas que desarrollan su actividad industrial o comercial dentro del sector metalúrgico en la provincia de Zaragoza, así como para prestarles apoyo y asesoramiento en el ámbito de sus actividades. La FEMZ

Scire. 8 : 2 (jul.-dic. 2002) 173-188. 
es la organización sectorial empresarial más importante de Aragón y la que cuenta con el mayor número de asociados, más de 2.000. Agrupa a diversas asociaciones profesionales, con personalidad jurídica propia y con la finalidad de la defensa de los intereses específicos de una determinada rama de actividad. De entre las más de cien actividades económicas que comprende la Federación, destacan por su importancia: Instalaciones Eléctricas; Talleres de Reparación de Automóviles; Fundición; Máquinas; Herramientas; Joyerías; Almacenistas de Aluminio; Fabricación, Componentes y Accesorios de Vehículos; Carpintería Metálica; Fabricación de Maquinaria Agrícola; Forja, Estampado, Embutición y Corte; Tratamiento y Recubrimiento de Metales; Calderería; Talleres Mecánicos Independientes; Electrónica; Relojeros; Almacenistas de Hierros; Reparación de TV, Radio y Electrodomésticos; Fabricantes de Joyería; y Comercio del Metal.

CEPYME Zaragoza y CEPYME Aragón, son organizaciones intersectoriales de ámbito provincial y autonómico respectivamente, que tienen como objeto fundamental el fomento, defensa y representación de los intereses de las Pequeñas y Medianas Empresas de su respectivo ámbito territorial. CEPYME Zaragoza, fue creada en el año 1978 y CEPYME ARAGÓN en el año 1989, integrando en la actualidad a las Confederaciones Provinciales CEPYME Huesca, CEPYME Teruel y CEPYME Zaragoza. En CEPYME Aragón están integradas un centenar de asociaciones empresariales de todos los sectores de la actividad productiva, que en su conjunto representan a más 20. 000 empresas. La Fundación "Cultura y Empresa" surgió en 1996 de la voluntad de CEPYME Zaragoza de ampliar y singularizar sus responsabilidades sociales. Su ámbito territorial de actuación es fundamentalmente la Comunidad Autónoma de Aragón. La Fundación tiene por objeto el desarrollo de todo tipo de actividades que contribuyan a impulsar el desarrollo empresarial y cultural de Aragón, promocionar la formación empresarial, así como la orientación y el perfeccionamiento profesional de empresarios, directivos y personal de empresas, además de la preparación para la inserción profesional.

\section{Los servicios de documentación en las organizaciones: piezas claves de sus sistemas de información}

\section{1. La información: necesidad y recurso para las organizaciones}

Toda organización necesita disponer de diferentes tipos de información. Para su correcto funcionamiento debe conocer claramente su situación financiera, sus recursos humanos (características de la plantilla, datos sobre contratación, formación, promoción de los trabajadores), su nivel de actividad o de producción, su situación comercial. Además de esta información interna, debe disponer de información externa, tanto del sector concreto en el que desarrolla su actividad (com-

Scire. $8: 2$ (jul.-dic. 2002) 173-188. 
petidores, clientes y proveedores) como del entorno general en el que se mueve (situación económica, legal, demográfica, tecnológica). Esta necesidad es máxima en las organizaciones empresariales, donde la información es la materia prima de su quehacer diario, ya que la mayoría de sus actividades son procesos informacionales. Así, para poder representar y defender los intereses del empresariado, las organizaciones deben estar perfectamente informadas de la realidad empresarial, de sus necesidades y aspiraciones, y así mismo deben tener un conocimiento profundo del entorno político, legislativo, económico, fiscal, laboral, social y tecnológico en el que se desarrolla la actividad empresarial de sus asociados.

$\mathrm{El}$ asesoramiento a las empresas en las distintas materias de su interés obliga a las organizaciones a disponer de la mejor y más actual información sobre dichas materias. Para conseguir la adecuación de los servicios prestados a las necesidades reales de las empresas es imprescindible conocer sus problemas, sus carencias, la oferta de servicios prestados por otras entidades públicas o privadas. Y finalmente, toda organización necesita disponer de información sobre su posición competitiva para enmarcar las acciones desarrolladas tanto por la propia organización como por sus competidores (Escobar Rodríguez, 2001). Carecer de información de calidad tiene repercusiones que pueden ir desde las molestias organizativas hasta la toma de decisiones mal fundamentadas o, incluso, el cese de sus actividades (Huang, Lee y Wang, 2000). Ello obliga a las organizaciones a dotarse de instrumentos que les permitan satisfacer sus necesidades informativas. Pero la información para una organización puede ser mucho más que una necesidad a satisfacer, ya que su adecuada explotación la convierte en un importante recurso generador de valor, y que, a diferencia de los recursos materiales, puede reutilizarse, compartirse, distribuirse, intercambiarse sin que se produzca pérdida de valor; de hecho, generalmente el valor se multiplica con su uso. Por ello, la información de calidad se reconoce de forma creciente como uno de los bienes más valiosos de una organización y en consecuencia tanto empresas como administraciones intentan dar con formas para aprovechar la información y los conocimientos; y la solución la encuentran en el establecimiento de sistemas de información.

\section{2. Las organizaciones como sistemas de información}

Los sistemas de información han sido definidos como el conjunto integrado de procesos, principalmente formales, desarrollados en un entorno usuario-ordenador, que, operando sobre un conjunto de datos estructurados de acuerdo con las necesidades de una organización, recopilan, elaboran y distribuyen selectivamente la información necesaria para la operatividad habitual de la organización y las actividades propias de la dirección en la misma (Ranguelov, 2000).

Conseguir el éxito de un sistema de información no es fácil, así recientes investigaciones han demostrado que hasta un $75 \%$ de los grandes sistemas de

Scire. $8: 2$ (jul.-dic. 2002) 173-188. 
información han fracasado desde el punto de vista operativo (Laudon \& Laudon, 1996; citado por Cornella, 2000, p. 80). ¿Cuáles pueden ser las causas de estos fracasos? En primer lugar, es evidente que gestionar información no es sencillo, pero esta no parece ser la principal causa de los fracasos. Generalmente, los sistemas de información se han creado para atender exclusivamente a las necesidades informativas de los directivos y, sin embargo, los estudios demuestran que éstos prefieren buscarse sus propias fuentes informales antes que acudir a los sistemas de información (Cornella, 2000, p. 91). Por otro lado, es corriente que los sistemas de información se conciban como sistemas superpuestos a las organizaciones, y con un elevado componente tecnológico, con fuertes inversiones en tecnologías de la información que facilitan el acceso y la transferencia a grandes cantidades de información. Estos sistemas se han centrado casi exclusivamente en las tecnologías, dejando en segundo lugar su objetivo fundamental: la información y las personas. Y ésta parece ser la principal causa de los fracasos, ya que las tecnologías, aún siendo muy necesarias, no son suficientes. Éstas pueden ayudar en las fases intermedias del ciclo de la información —almacenamiento, síntesis y transmisión-, pero no sirve de mucho a la hora de generarla o utilizarla, pues, en último término, la gestión de la información es una tarea esencialmente humana (Davenport, 1999).

Un sistema de información tiene que plantearse como objetivo la explotación inteligente de la información, y es precisamente la utilización de la información por los usuarios la que determina el éxito o el fracaso del sistema. ¿Esto que implica? En primer lugar, que la información es el objeto de un sistema de información. El énfasis se debe poner en la calidad de la información y en su aprovechamiento y no en el sistema informático. Como indican Huang, Lee y Wang (2000) la información debe tratarse como un producto final que debe satisfacer las necesidades del consumidor, habiendo claras analogías entre la fabricación de un producto y la generación de información. Este proceso de generación y utilización de la información debe partir del conocimiento de las necesidades informativas de la organización, para en consecuencia recoger los datos procedentes de una amplia variedad de fuentes informativas. Una vez captados los datos, deben ser analizados y procesados para generar los productos informativos que ya finalmente podrán ser difundidos (Timmerman, 2000).

En segundo lugar, el sistema de información debe tener en cuenta que las personas son el eje de un sistema de información. No tiene mucho sentido que una organización invierta en adquirir o generar información y en diseñar, desarrollar, instalar y mantener sistemas, si sus miembros no utilizan la información que reciben (Cornella, 2000). Y hay que tener en cuenta que en una organización todo su personal - y no exclusivamente los cargos directivos- necesitan disponer de información para el correcto desempeño de sus funciones. Evidentemente las necesidades informativas variarán según las funciones y competencias de

Scire. $8: 2$ (jul.-dic. 2002) 173-188. 
cada miembro, pero todos ellos son potenciales usuarios de un sistema de información. Por otra parte, el personal de una organización es tanto consumidor como generador de información sobre su ámbito de trabajo, por lo deben estar capacitados para gestionar su propia información, para crear sus propios sistemas personales de información (2). Y es que, como señala Timmerman (2000, p. 18), en general la causa de los problemas no está en los sistemas de información, sino en las personas que los usan. A su vez, las organizaciones deberán desarrollar estrategias que les permitan aprovecharse de toda la información generada por todos sus trabajadores, deben ser capaces de integrar todos los sistemas personales para constituir un sistema de información corporativo del que formen parte y a su vez se sirvan todo el personal de la organización. En definitiva, tal y como señala Cornella, el sistema de información de una organización debería entenderse como un sumatorio de los sistemas de información de sus miembros (2000, p. 25), por lo que debemos superar el discurso sobre los sistemas de información en las organizaciones, para entrar de pleno en el discurso sobre las organizaciones como sistemas de información (ibidem, p. IX).

\section{3. La documentación en un sistema de información}

Aunque buena parte de la información circula por mecanismos informales - conversaciones en los pasillos, comentarios, rumores-, lo cierto es que la mayoría de los datos e informaciones que maneja una organización proceden de documentos de variada tipología (3). Por lo tanto, para conseguir una buena gestión de la información es imprescindible organizar y conseguir un buen sistema de gestión documental. A grandes rasgos, en una organización podemos diferenciar dos tipos de documentación:1) la documentación generada en el desempeño de sus funciones, como plasmación de las actividades realizadas; 2) las distintas publicaciones o fuentes de información que reciben o incluso elaboran ellas mismas: libros, revistas, prensa, literatura gris, vídeos, bases de datos, Internet. El primer tipo de documentación formará el archivo de la organización y el segundo la biblioteca o centro de documentación. Por lo tanto, el sistema de gestión documental de una organización lo conformarán el archivo y la biblioteca o centro de documentación de dicha organización, y estos en consecuencia son los auténticos centros neurálgicos de su sistema de información.

\section{Planificación del Servicio de Documentación y Archivos}

La puesta en marcha de un nuevo servicio debe estar perfectamente planificada. La planificación partirá de una etapa previa analítica que permita conocer la situación y evaluar las necesidades, seguida de una propuesta de actuación, que, de ser aprobada, pasará a ponerse en práctica. Y este fue el proceso seguido con el Servicio de Documentación y Archivos que se presenta.

Scire. $8: 2$ (jul.-dic. 2002) 173-188. 
Tras analizar las características fundamentales de las organizaciones en las que se iba enclavar el servicio (estructura organizativa, funciones y actividades realizadas, historia y evolución institucional) así como de su documentación (tipologías, volumen, métodos de organización, instalación y conservación de la documentación), se llegó a unas conclusiones generales que nos permitieron diagnosticar la situación y elaborar un proyecto de actuación. El proyecto bajo el título de Sistema de Información de las Organizaciones Empresariales CEPYME ARAGÓN, CEPYME ZARAGOZA, Federación de Empresarios del Metal de Zaragoza y Fundación "Cultura y Empresa": Proyecto para su implantación fue presentado a la dirección de estas organizaciones para su aprobación. El proyecto señalaba como objetivo final el establecimiento de un Sistema de Información que ofreciese a los directivos y a los empleados de las organizaciones empresariales una información efectiva y de calidad que les facilitase la toma de decisiones, el diseño de sus estrategias, agilizase la gestión administrativa y la resolución de los asuntos. En dicho sistema de información, el Servicio de Documentación y Archivos ocuparía un lugar central. Este servicio estaría conformado por dos unidades. En primer lugar, el archivo, encargado de organizar y custodiar toda la documentación generada o recibida por las organizaciones en el ejercicio de sus actividades directivas, administrativas, económicas y asesoras, es decir su información interna. En segundo lugar, la biblioteca especializada o centro de documentación: encargado de seleccionar, adquirir, tratar y difundir las fuentes de información externas necesarias para el desarrollo de sus funciones. A partir de la división funcional del Servicio, en el proyecto se detallaban las fases a seguir para su puesta en funcionamiento, los procedimientos y procesos a realizar en cada fase, el personal implicado en ellas, y los recursos materiales y humanos precisos. Asimismo, se incluyó una temporalización con los plazos previsibles para cada una de las fases.

\section{La puesta en marcha}

\section{1. Una puesta en marcha escalable}

El primer problema con el que se encuentra un servicio de nueva creación es el de la credibilidad interna. Conseguir que la dirección avale y haga propios los objetivos marcados en el proyecto de implantación del servicio es un reto del que va a depender su funcionamiento futuro. Los obstáculos a los que a los que al principio se debe enfrentar un servicio de Ddocumentación en el ámbito empresarial para lograr esta credibilidad interna son, en primer lugar, el desconocimiento de las ventajas que ofrece un eficaz servicio de documentación en una organización, y la escasez de referentes para mostrar como ejemplos de funcionamiento de un servicio de documentación en el ámbito empresarial. 
En consecuencia los directivos generalmente no son capaces de valorar $a$ priori lo que aportará la implantación de este nuevo servicio, por lo que sólo su puesta en funcionamiento y la consecución de objetivos es la que va a demostrar su valía. Y este es otro de los obstáculos a salvar. En un entorno empresarial marcado por la inmediatez, el servicio de documentación necesita de tiempo para conseguir sus objetivos, máxime si los recursos personales con los que se cuentan son limitados. Por ello, la puesta en funcionamiento de un servicio de documentación debe ser escalable, marcándonos objetivos a corto y medio plazo, que hagan visible nuestro trabajo y permitan valorar su utilidad.

\section{2. La necesaria implicación de todo el personal}

Evidentemente, un servicio de documentación y archivos en una organización empresarial necesita de documentalistas para poder llevar a cabo sus cometidos. Pero junto a los documentalistas es imprescindible la colaboración de otros profesionales. Entre estos se debe destacar a los informáticos. Actualmente es impensable un servicio de documentación sin un adecuado soporte tecnológico. Buena parte de los documentos objeto de nuestro quehacer diario, la mayoría de los procesos que realizamos, así como los canales a través de los que distribuimos y facilitamos el acceso a la información están en un entorno informático. Por ello, la estrecha colaboración con el personal informático resulta imprescindible.

En tanto que el servicio se plantea como objetivo el potenciar el acceso y difusión de la información, resulta muy enriquecedora la colaboración con los profesionales de la comunicación, quienes nos pueden aportar sus conocimientos sobre la situación informativa y comunicativa de la organización: cauces naturales por los que fluye la información, las barreras internas que se establecen, las áreas más transparentes y las más opacas.

La colaboración con las personas que tienen encomendadas una función asesora en alguna de las materias de interés empresarial — tecnología, medio ambiente, relaciones laborales, fiscalidad, financiación, cooperación empresarial, relaciones internacionales, etc.— resulta fundamental. Estas personas además de asesorar a las empresas asociadas suelen ser los representantes de las organizaciones empresariales en las comisiones técnicas de distintas instituciones públicas o privadas. Por lo tanto, conocen las necesidades informativas a las que deben atender las organizaciones empresariales, conocen las principales fuentes de información sobre las materias en las que están especializados, y son los receptores de la información más actualizada sobre estas materias. Todas ellas tienen desarrolladas sus propios sistemas personales de información, por lo que es necesario conocer como funcionan, para colaborar en su mejora en los casos que sea necesario y para intentar integrarlos en el sistema de información corporativo.

Scire. $8: 2$ (jul.-dic. 2002) 173-188. 
En una organización empresarial todo su personal, desde los auxiliares hasta los directivos, realiza tareas documentales. Es por ello que necesitamos contar con la colaboración de todo el personal, en un principio para que nos aporten información sobre los procesos documentales que llevan a cabo y finalmente para que adopten todas las innovaciones necesarias para mejorar la gestión documental y potenciar el intercambio de la información. Conseguir esta colaboración es otro de los retos que deben ser abordados. Para ello tendremos que superar la resistencia al cambio de parte del personal, por lo que será necesario contar con el apoyo de la dirección, que es quien tiene la capacidad para "imponer" los cambios. Pero el proceso tiene que ser de convicción más que de imposición por lo que es necesario mantener perfectamente informado a todo el personal de los objetivos perseguidos, concienciándoles de las ventajas que les reportará las innovaciones. Por otra parte, si el personal no ha conocido experiencias anteriores, resulta muy difícil que adopten de buen grado las nuevas prácticas. Por ello, es bueno acometer experiencias piloto que permitan a todo el personal conocer las ventajas de las innovaciones.

\section{3. La metodología}

El funcionamiento del Servicio de Documentación y Archivos se plasma en la realización de una serie de procesos técnicos, desarrollados sobre una plataforma tecnológica.

\section{3. 1. La plataforma tecnológica}

Dado que el fin último del servicio es contribuir al desarrollo de un sistema de información corporativo, su plataforma tecnológica debe estar plenamente integrada en la estructura interna de las organizaciones. Las tecnologías de groupware o trabajo en grupo - en cuanto que permiten a grupos de personas trabajar juntas electrónicamente, facilitan la comunicación, la colaboración y la captura del conocimiento de las organizaciones - son las que se adecuan a la finalidad del servicio. El soporte tecnológico de estas funciones lo constituyen las aplicaciones informáticas de mensajería electrónica, las bases de datos compartidas y los sistemas de flujo de trabajo. En nuestro caso, la práctica totalidad de las bases de datos elaboradas por los departamentos de las organizaciones empresariales así como las comunicaciones internas se realizan a través de Lotus Notes, un programa que reúne una plataforma de gestión documental, una infraestructura de mensajería, un entorno para el desarrollo de aplicaciones de flujo de trabajo (workflow) y que permite una integración total con Internet. Por todo ello, se optó por el desarrollo de las bases de datos del Servicio de Documentación y Archivos sobre Lotus Notes.

\section{3. 2. Los procesos técnicos}

Como señala García Montero, aunque la plataforma tecnológica tiene gran importancia, más importantes son los aspectos organizativos, es decir, una buena 
definición de los procedimientos que se van a realizar (García Montero, 1998, p. 19). Para conseguir la mayor calidad de los procesos documentales el marco metodológico más adecuado es el establecido por las ciencias y técnicas de la documentación, las cuales nos ofrecen un elevado grado de normalización de los procesos, a la par que el necesario bagaje teórico y conceptual para poder enmarcar nuestra actuación. La existencia de un cuerpo doctrinal y metodológico fuertemente consolidado y ampliamente aceptado, nos evita tener que "inventar" nuevas soluciones a problemas ya resueltos. Lo cual no quiere decir que debamos estancarnos en unos saberes fosilizados; muy al contrario, debemos evolucionar al ritmo de las nuevas situaciones, y dar soluciones a los nuevos problemas que constantemente surgen; pero en este empeño afortunadamente tenemos una base muy firme sobre la que evolucionar. En nuestro caso, la distinta naturaleza de los documentos objeto del Servicio de Documentación y Archivos han hecho necesaria la aplicación de las metodologías especificas de las distintas disciplinas documentales dependiendo del tipo de documentación tratado, la Archivística para la documentación de archivo, y la Biblioteconomía y Documentación para las fuentes de información externas. Todo ello sin olvidar los puntos confluyentes que tienen las distintas disciplinas y los aportes mutuos que se pueden establecer entre ellas.

En líneas generales, la metodología archivística parte de la necesidad de controlar la documentación generada por una institución en el desempeño de sus funciones desde el momento que se produce hasta que es eliminada o conservada permanentemente. Para ello es necesario la existencia de distintos tipos de archivos que recojan secuencialmente la documentación a lo largo de su ciclo vital, y que tendrán encomendadas distintas funciones en virtud de la edad de la documentación. El tratamiento archivístico se basa en el respeto del contexto en el que se producen los documentos, el cual debe respetarse a lo largo de todo su ciclo vital, especialmente en su primera fase, la de su producción. Los documentos de archivo no pueden entenderse aisladamente como meros soportes de información, sino que deben ir ligados al procedimiento administrativo del que forman parte. Por ello, es necesario asegurar la unidad de los expedientes, y de ese cometido se ocupan los archivos de oficina. Los documentos de archivo se producen secuencialmente al ritmo de la actividad de un órgano productor en el desempeño de sus funciones. Este carácter seriado de la documentación y el respeto al contexto orgánico funcional en el que surgen será la base de su clasificación, ordenación y descripción. Esta metodología es válida independientemente del soporte de la documentación, y por lo tanto debe ser aplicada tanto a la documentación en papel como a la producida en soporte digital.

Por su parte, formar y mantener una colección de fuentes de información, organizarla debidamente en función de los usuarios y ponerla a su disposición son en esencia las funciones que debe desarrollar una biblioteca o centro de docu-

Scire. 8 : 2 (jul.-dic. 2002) 173-188. 
mentación. La satisfacción de las necesidades informativas de los usuarios es la razón de ser de estos centros. Para ello, se deben conocer previamente las necesidades de los usuarios, para en consecuencia buscar, localizar, seleccionar y, en su caso, adquirir aquellas fuentes de información que satisfagan esas necesidades.

La tipología de estas fuentes de información es variada —enciclopedias, diccionarios, manuales, tratados, monografías, publicaciones periódicas, publicaciones oficiales, anuarios, directorios, guías, documentación estadística, literatura gris- y los soportes irán desde el papel al CD-ROM, sin olvidar Internet. Para posibilitar el almacenamiento, recuperación, utilización y difusión de estas fuentes de información se lleva a cabo el análisis documental, que englobará tanto el análisis formal como el análisis de contenido (clasificación, indización y resumen).

\section{Las actuaciones realizadas}

\section{1. Actuación archivística}

Las actuaciones archivísticas llevadas a cabo partieron del establecimiento de los distintos tipos de archivos que conforman el sistema archivístico de las organizaciones empresariales: los archivos de oficina localizados en los despachos del personal directivo, técnico y administrativo, y en los que se conserva la documentación en trámite y de uso muy frecuente; y los archivos generales donde se conserva la documentación sin vigencia administrativa y que haya sido transferida desde los archivos de oficina, y donde permanecerá mientras perduren algunos de sus valores administrativos, jurídicos, legales, informativos o históricos. La identificación de las series documentales generadas por las cuatro organizaciones empresariales ha sido uno de los primeros procesos llevados a cabo. Mediante este proceso hemos conocido la estructura organizativa de cada una de las organizaciones, las competencias, funciones y actividades que han desarrolladas a lo largo de los años y la evolución que han seguido (4). A partir de este conocimiento se elaboraron los cuatro cuadros clasificatorios de la documentación, unos cuadros que se han ido perfeccionando conforme se ha profundizado en la organización y descripción de la documentación, el siguiente proceso llevado a cabo. En la descripción de la documentación, se sigue la reciente normativa internacional de descripción archivística ISAD(G). Se utiliza como soporte para este proceso una base de datos - todavía en desarrollo- que recoge la descripción de las unidades documentales y en la que se irán incorporando los módulos para la descripción del resto de niveles de descripción. En estos momentos se está finalizando la organización y descripción de la documentación albergada en los archivos generales.

Por lo que respecta a los archivos de oficina, se está desarrollando una experiencia piloto, de gran calado por afectar a uno de los órganos centrales de la gestión documental de una organización como es el registro de correspondencia. La 
actuación ha consistido en la transformación y modernización de los procesos llevados a cabo por este departamento. Así se ha procedido a la informatización de los registros de entrada y salida de correspondencia, mediante la creación de cuatro bases de datos — una por organización— en la que además de los habituales campos descriptivos de los registros de correspondencia — número y fecha de entrada o salida, datos del remitente, datos del destinatario, fecha del documento recibido o enviado, y resumen de su contenido- contiene unos campos para la clasificación de la documentación de acuerdo a los cuadros clasificatorios establecidos para las organizaciones. De este modo toda la documentación recibida o enviada queda clasificada y descrita de acuerdo a los criterios establecidos desde el Servicio de Documentación y Archivos. Además a estas bases de datos se les anexan las imágenes de los documentos previamente digitalizados, los cuales pueden ser consultados desde los distintos ordenadores conectados a la red informática de las organizaciones, de acuerdo a los perfiles de los usuarios. De esta manera se consigue un mejor y más rápido acceso a la documentación, se reduce de manera sustancial la duplicación de documentos en soporte papel con el consiguiente ahorro en el espacio necesario para su conservación, y se posibilita un mejor control de la documentación original.

\section{2. Actuación bibliotecaria}

El modelo de biblioteca establecido es descentralizado. Así, para posibilitar que los departamentos tengan inmediatamente accesibles las fuentes de información que tratan sobre sus temas de interés, cada uno de ellos cuenta con su propia "biblioteca departamental". Para controlar todas estas fuentes de información descentralizadas y favorecer el conocimiento y acceso de todo el personal a las mismas se ha elaborado una base de datos sobre Lotus Notes, instalada en el servidor central y a la que tiene acceso todo el personal desde su propio ordenador.

La base de datos cuenta con varios módulos. Un módulo de catalogación que incluye un campo para señalar la clase de documento, campos para la descripción bibliográfica (se corresponde con las áreas establecidas en las reglas de catalogación), para los puntos de acceso (autor personal, entidad, título uniforme, nombre de congreso), para señalar la ubicación (departamento y signatura), para la clasificación e indización, un campo para incluir el índice de la publicación y otro para el resumen. El segundo módulo automatiza el vaciado de los artículos de revista, y los campos que contiene son: autor, título, publicación, número y fecha, páginas, resumen y palabras clave. Un tercer módulo es el de registro con unos campos descriptivos básicos, datos de registro (número y fecha de ingreso), campos para las condiciones de adquisición (proveedor, dirección, teléfono, fax, correo electrónico, precio, departamento destinatario), y campos para el control de las publicaciones periódicas (periodicidad, fecha de inicio de la publicación, y

Scire. $8: 2$ (jul.-dic. 2002) 173-188. 
números recibidos). Y un cuarto módulo, desde el que los usuarios pueden solicitar directamente el préstamo de publicaciones, la reproducción de artículos o la satisfacción de consultas de información generales.

En estos momentos se está finalizando la catalogación retrospectiva de las publicaciones que se habían ido acumulando a lo largo de los años en los distintos departamentos. Además, gracias a la colaboración de todo el personal, todas las nuevas adquisiciones una vez que ingresan en la sede de las organizaciones empresariales pasan al Servicio de Documentación, donde se procede a su registro, catalogación, clasificación y, finalmente, a su ordenación en la correspondiente biblioteca departamental y a la difusión de su información. Desde el Servicio además de posibilitar el acceso a la información contenida en la base de datos, se lleva a cabo distintas actividades de difusión de la información. Así se elaboran dos boletines de periodicidad trimestral: el Boletín de novedades bibliográficos —que informa de las publicaciones que han ingresado en el último trimestre en los distintos departamentos- y el Boletín de artículos de revistas que informa de los últimos artículos contenidos en una serie de revistas técnicas-. A partir del conocimiento de los intereses del personal se realizan actividades de difusión selectiva de la información. Así, mensualmente se elabora el boletín Nuestra Selección, en el que se recoge una selección artículos en virtud de su potencial interés. Además se llevan a cabo "alertas informativas", informando de manera personalizada y en el tiempo más breve posible de las novedades sobre los temas de su interés. Los boletines y las alertas informativas se distribuyen a través del correo electrónico interno de Lotus Notes. Además para mantener informadas a las empresas asociadas, los Boletines se publican en el portal www.conectapyme.com, y en las páginas web de la FEMZ (www. femz.es) y de la Fundación Cultura y Empresa (www.culturayempresa.es).

\section{3. Colaboración en proyectos en Internet}

El Servicio de Documentación y Archivos también colabora en distintos proyectos que se desarrollan en Internet. Así, ha participado en la creación de la página web de la Fundación "Cultura y Empresa", encargándose de la estructuración de la información y del contenido de uno de sus apartados, el correspondiente a "Fundaciones". Y participa activamente en el Portal conectapyme.com, una idea y un reto de la Confederación de la Pequeña y Mediana Empresa Aragonesa (CEPYME-ARAGON) en su apuesta por mantener un espíritu de innovación constante, apuntarse a las vanguardias tecnológicas y fomentar el uso de las nuevas tecnologías entre las pequeñas y medianas empresas. El cometido del Servicio de Documentación en este portal consiste en la estructuración de la información. 


\section{Notas}

(1) La libertad de constitución de las organizaciones empresariales se estableció en España por la ley 19/1977, de 1 de abril, reguladora del derecho de asociación sindical, desarrollada por el decreto 873/1977, de 22 de abril, siendo reconocidas en el artículo 7 de la Constitución Española como entidades para la defensa y promoción de los intereses económicos y sociales que les son propios.

(2) En una sociedad intensiva en información es necesario que todo el personal disponga de una serie de habilidades informacionales, como navegar por las distintas fuentes de información, utilizar los sistemas de información, discriminar la calidad de las fuentes de información, determinar la fiabilidad de las fuentes, dominar la sobrecarga de información, aplicar la información a problemas reales, saber comunicar la información a otros (Cornella, 2000, p. 48-49)

(3) Según Gary Cleveland, el 90\% de la información de una organización reside en documentos. Citado por Martínez Sánchez (1997, p. 238)

(4) Las fuentes utilizadas para este proceso han sido: los organigramas de las organizaciones, las memorias de actividades, los boletines informativos propios, los folletos divulgativos de los servicios prestados, las circulares enviadas a los asociados, las actas de las reuniones de sus órganos de gobierno, la información aportada en las respectivas páginas web de estas organizaciones y las charlas con el personal.

\section{Referencias}

Arias Coello, Alicia; Portella Filgueiras, Isabel (1997). Sistemas de información y sistema de calidad: relación y dependencia en las organizaciones empresariales. // Documentación de las ciencias de la información. 20 (1997) 11-20.

Bustelo Ruesta, Carlota (2000). Gestión documental en las empresas: una aproximación práctica. // Actas de las VII Jornadas Españolas de Documentación FESABID 2000. 189-195.

Cornella, Alfons (2000). Infonomía.com: la empresa es información. Bilbao: Deusto, 2000.

Davenport, Thomas H (1999). El punto sobre la I de tecnología de la información. // Expansión. Negocios en la era digital. (30 sept. 1999). 4-6.

Earl, Michael J. (1999). Todas las empresas son empresas de información. // Expansión, Negocios en la era digital. (7 oct. 1999) 5-7.

Escobar Rodríguez, Tomás (2001). Reflexiones en torno a la información necesaria para el desarrollo de la gestión directiva. // Alta Dirección. 216 (2001) 9-24.

García Montero, María Antonia (1998). Groupware y gestión de documentos en la empresa. // Revista General de Información y Documentación. 2 (1998) 11-30.

Herranz Palo, María (2001). La gestión documental. // Partida Doble. 124 (jul.-ag. 2001) 36-43.

Huang, Kuan-Tsae; Lee, Yang W.; Wang, Richard Y. (2000). Calidad de la información y gestión del conocimiento. Madrid: AENOR, 2000.

Las organizaciones empresariales al servicio de las empresas. // Conclusiones y ponencias de la Conferencia Empresarial Nuevos Tiempos, nuevos retos: 22-23 mayo de 2000. Madrid: CEOE, 2000. 207-246.

Scire. $8: 2$ (jul.-dic. 2002) 173-188. 
Martínez Sánchez, José Manuel; Hilera Sánchez, José Ramón (1997). Los sistemas de gestión documental en el ámbito del trabajo corporativo. // Documentación de las ciencias de la información. 7 (1997) 237-255.

Núñez Fernández, Eduardo (1999). Organización y gestión de archivos. Gijón: Trea, 1999.

Orellana Moraleda, Concepción (2001). Implantación de sistemas de información: el cambio cultural, importancia de la formación y la información. // Alta Dirección. 216 (2001) 86-92.

Paños Álvarez, Antonio (1999). Reflexiones sobre el papel de la información como recurso competitivo de la empresa. // Anales de documentación. 2 (1999) 21-38.

Ranguelov Youlianov, Stanislav (2000). Sistemas para gestionar la información organizativa y el conocimiento. URL: <http://www. gestiondelconocimiento.com/documentos2/stanislav_ranguelov/sistemas.htm>.

Timmerman, Frederick W. (2000). Cómo ganar el desafío de la información. Bilbao: Deusto, 2000. 